\title{
FREQUENCY LOCKING IN COUNTABLE CELLULAR SYSTEMS, LOCALIZATION OF (ASYMPTOTIC) QUASI-PERIODIC SOLUTIONS OF AUTONOMOUS DIFFERENTIAL SYSTEMS*
}

\author{
LAURENT GAUBERT ${ }^{\dagger}$
}

\begin{abstract}
We address the question of frequency locking in coupled differential systems and of the existence of some quasi-periodic solutions of a certain kind of differential systems. Those systems are named "cellular systems" quite generally as they deal with countable numbers of coupled systems in some general Banach spaces. Moreover, the inner dynamics of each subsystem does not have to be specified. We reach some general results about how the frequency locking phenomenon is related to the structure of the coupling map. Those results can be restated in terms of localization of a certain type of quasi-periodic solution of differential systems that may be seen as cellular systems. This paper gives some explanations about how and why synchronized behaviors naturally occur in a wide variety of complex systems.
\end{abstract}

Key words. coupled systems, synchronization, frequency locking, quasi-periodic solution

AMS subject classifications. 34D06, 34C25, 34C29

DOI. $10.1137 / 070700814$

1. Introduction. Synchronization is an extremely important and interesting emergent property of complex systems. The first example found in the literature goes back to the 17th century with Huygens' work [11],(see also [2]. This kind of emergent behavior can be found in artificial systems as well as in natural ones and at many scales (from individual cells to whole ecological systems). Biology abounds with periodic and synchronized phenomena, and the work of Prigogine shows that such behaviors arise within specific conditions: a dissipative structure generally associated with a nonlinear dynamics [20]. Biological systems are open, evolve far from thermodynamic equilibrium, and are subject to numerous regulating processes, leading to highly nonlinear dynamics. Therefore periodic behaviors appear (with or without synchronization) at any scale [21]. More generally, life itself is governed by circadian rhythms [9]. Those phenomena are as much attractive as they are often spectacular: from cicada populations that appear spontaneously every ten or thirteen years [10] or networks of heart cells that beat together [17] to huge swarms in which fireflies, gathered in the same tree, flash simultaneously [3]. This synchronization phenomenon occupies a privileged position among emergent collective phenomena because of its various applications in, e.g., neuroscience, ecology, and earth science, [28, 26, 16], as well as in the field of coupled dynamical systems, especially through the notion of chaotic systems' synchronization [18, 7] and the study of coupled oscillators [13]. This wide source of examples leads the field to be highly interdisciplinary, from pure theory to concrete applications and experiments.

The classical concept of synchronization is related to the locking of the basic frequencies and instantaneous phases of regular oscillations. One of the most successful attempts to explore this emergent property is due to Kuramoto [14, 15]. As in Kuramoto's work, these questions are usually addressed by studying specific kinds

\footnotetext{
${ }^{*}$ Received by the editors August 22, 2007; accepted for publication (in revised form) October 8, 2010; published electronically January 4, 2011.

http://www.siam.org/journals/siap/71-1/70081.html

†Centre Européen de Réalité Virtuelle, Laboratoire d'Informatique et des Systèmes Complexes, EA3883 UBO/ENIB, 25 rue Claude Chappe, 29280 Plouzané, France (gaubert@enib.fr).
} 
of coupled systems (see, for instance, [5, 22, 8]). Using all the classical methods available in the field of dynamical systems, researchers study specific trajectories of those systems in order to get information on possible attracting synchronized states $[29,13,22,19,8,12]$.

The starting point of this work initiated in [6] was the following question: "Why is synchronization such a widely present phenomenon?" In order to give some mathematical answer to this question, the first step is to build a model of coupled systems that is biologically inspired. This is done in the second section: after describing some basic material, we define the terms cellular systems and cellular coupler. If one would summarize the specificities of a cellular system, one could say that each cell (subsystem) of a cellular system receives information from the whole population (the coupled system) according to some constraints:

- a cell has access to linear transformations of all the others cells' states;

- the way this information is gathered depends (not linearly) on each cells' state itself.

In other words, a cell interprets its own environment via the states of the whole population and according to its own state.

It's a bit surprising that, despite this model's arising very naturally, it gives a good framework for addressing the main question. Indeed, in the third section we describe a localization result concerning some periodic and asymptotically periodic trajectories of cellular systems. It exhibits some links between the coupler's properties and the structure of periodic trajectories. This result insures that, in most cases, frequencies are locked.

The fourth section gives some example of applications to classical systems, as well as some general results that may be proved using this technique.

Moreover, as synchronization is strongly related to the more abstract field of dynamical systems, some results go out of the scope of coupled systems. If one thinks about the presence of attractors in a differential system, one may, for example, classify those as one of the following:

- point attractor,

- limit cycle,

- limit torus.

Those attractors can be related to coupled systems in an obvious way: roughly speaking, a point attractor may be seen as a solution of coupled systems for which each of the subsystems has a constant behavior. Similarly, a limit cycle may be thought of as the situation in which every subsystem oscillates, all frequencies among the whole system being locked. A limit torus is a similar situation which differs from the previous one by the fact that the frequencies are not locked (noncommensurable periods of a quasi-periodic solution of the whole coupled system). Hence, the three previous cases may be translated into the coupled dynamical systems context as follows:

- point attractor $\leftrightarrow$ constant trajectories;

- limit cycle $\leftrightarrow$ periodic trajectories, locked frequencies;

- limit torus $\leftrightarrow$ periodic trajectories, unlocked frequencies.

Therefore, we deduce some results about the localization of solutions of the third type, quasi-periodic solutions, using the point of view of coupled dynamical systems. The results of this fourth section may help us to understand why the second case is the most observed in natural systems, which may be seen as coupled dynamical systems (many levels). Indeed, the section shows how the cellular systems point of view may be applied to a wide class of differential systems in order to address some of those questions with algebraic tools (as our results rule out the existence, in most 
cases, of solutions with noncommensurable periodic coordinates).

2. Basic material and notation. As our model is inspired by cellular tissues, some terms clearly come from the vocabulary used to describe those kinds of complex systems.

2.1. Model of population behavior. Here are the basics of our model.

A population $\mathcal{I}$ is a countable set, so we may consider it as a subset $\mathcal{I} \subset \mathbb{N}$. Moreover, only the cardinality of $\mathcal{I}$ matters, so $\mathcal{I}$ may be chosen as an interval of integers. Elements of $\mathcal{I}$ are called cells.

We suppose that the systems we want to study are valued in some Banach spaces. Thus, for any $i \in \mathcal{I},\left(E_{i},\|.\|_{i}\right)$ is a Banach space, and the state space of $\mathcal{I}$ is the vector space $\mathcal{S}=\prod_{i \in I} E_{i}$.

We will sometimes identify $E_{i}$ with

$$
\prod_{j<i}\{0\} \times E_{i} \times \prod_{j>i}\{0\} \subset \mathcal{S}
$$

and then consider it as a subspace of $\mathcal{S}$.

Moreover, $\mathcal{S}$ has the natural structure of a module on $\mathbb{R}^{\mathcal{I}}$; given $\lambda: \mathcal{I} \rightarrow \mathbb{R}$ and $x \in \mathcal{S}$, one may define $\lambda . x$ as

$$
\lambda \cdot x=\left(\lambda(i) \cdot x_{i}\right)_{i \in \mathcal{I}} .
$$

We denote by $\mathcal{S}_{\infty}$ the space of uniformly bounded states:

$$
\mathcal{S}_{\infty}=\left\{x \in \mathcal{S}, \sup _{i \in \mathcal{I}}\left\|x_{i}\right\|_{i}<\infty\right\} .
$$

This subspace will sometimes be useful as, embodied with the norm $\|x\|_{\infty}=\sup _{i \in \mathcal{I}}\left\|x_{i}\right\|_{i}$, it's a Banach space, allowing the classic Picard-Lindelöf theorem to be valid.

Given an interval $\Omega \subset \mathbb{R}$, a trajectory $x$ of $\mathcal{I}$ is an element of $\mathcal{C}^{\infty}(\Omega, \mathcal{S})$. Such an $x$ is then described by a family of smooth applications $\left(x_{i}\right)_{i \in \mathcal{I}}$ such that $\forall i \in \mathcal{I}$

$$
\begin{aligned}
x_{i}: \Omega & \longrightarrow E_{i}, \\
t & \longmapsto x_{i}(t) .
\end{aligned}
$$

The space of trajectories on $I$ is denoted by $\mathcal{T}$.

Each cell $i$ is supposed to behave according to an autonomous differential system given by a vector field $F_{i}: E_{i} \rightarrow E_{i}$. Thus, given a family of functions $\left\{F_{i}\right\}_{i \in \mathcal{I}}$, we define the vector field $F_{\mathcal{I}}$ on $\mathcal{S}$ as

$$
\begin{aligned}
F_{\mathcal{I}}: \mathcal{S} & \longrightarrow \mathcal{S}, \\
x & \longmapsto F_{\mathcal{I}}(x),
\end{aligned}
$$

where, for any $i \in \mathcal{I}$,

$$
\left[F_{\mathcal{I}}(x)\right]_{i}=F_{i}\left(x_{i}\right) .
$$

A period on $\mathcal{I}$ is a map $\tau: \mathcal{I} \rightarrow \mathbb{R}_{+}^{*}$. A trajectory $x \in \mathcal{T}$ is said to be component $\tau$-periodic $(\mathrm{CP}(\tau))$ if, for any $i \in \mathcal{I}, x_{i}$ is $\tau(i)$-periodic and nonconstant. In that case, $\tau(i)$ is a period of the cell $i$. The space of such trajectories is written $\mathcal{T}_{\tau}$. If $\tau$ is bounded, a trajectory in $\mathcal{T}_{\tau}$ that is not globally periodic is said to be component $\tau$-quasi-periodic $(\mathrm{CQP}(\tau))$. 


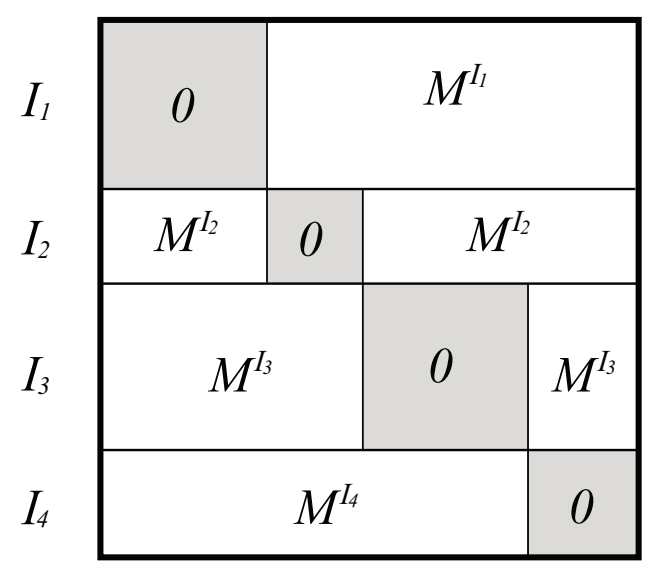

FIG. 2.1. Projection of a matrix according to a partition of $\mathcal{I}$.

A trajectory $x$ is said to be asymptotically component $\tau$-periodic $(\operatorname{aCP}(\tau))$ if there exist some $y$ which is $\mathrm{CP}(\tau)$ and $\alpha$ which vanishes when $t \rightarrow+\infty$ such that

$$
x=y+\alpha .
$$

In a similar way we define an asymptotically component $\tau$-quasi-periodic trajectory $(\operatorname{aCQP}(\tau))$.

Remark 1 . We stress the point that a period of a component periodic trajectory need not be a minimal period $(\tau(i)$ isn't necessarily a generator of the group of periods of $x_{i}$ ). Nevertheless, the definition of $\mathcal{T}_{\tau}$ avoids any trajectory which contains some constant component (none of the $x_{i}$ can be a constant map), as they may be seen as degenerate (localized into a "hyperplane" of $\mathcal{S}$ ).

We recall that a (finite) subset $\left\{\tau_{1}, \ldots, \tau_{k}\right\}$ of $\mathbb{R}$ is said to be rationally dependent if there exist some integers $l_{1}, \ldots, l_{k}$ not all zero and such that

$$
l_{1} \tau_{1}+\cdots+l_{k} \tau_{k}=0
$$

Then there exists a unique lowest common multiple $(\mathrm{lcm}) \tau_{0}$ for which there exists $n_{1}, \ldots, n_{k}$ such that

$$
n_{1} \tau_{1}=\cdots=n_{k} \tau_{k}=\tau_{0}
$$

An infinite set of real numbers is said to be rationally dependent if any finite subset is rationally dependent.

Now, any period $\tau$ on $\mathcal{I}$ defines a equivalence relation on $\mathcal{I}$ as

$$
i \sim j \Leftrightarrow_{\tau}\{\tau(i), \tau(j)\} \text { is a dependent set. }
$$

Hence we may consider the partition $\mathcal{I}(\tau)$ of $\mathcal{I}$ into equivalence classes ( $K$ countable):

$$
\mathcal{I} / \tau=\left\{\mathcal{I}_{k}\right\}_{k \in K} .
$$

Let $M=\left(m_{i j}\right)_{(i, j) \in \mathcal{I}^{2}}$ be a matrix indexed on $\mathcal{I}^{2}$; if $J=\left\{I_{1}, \ldots, I_{K}\right\}$ is a partition of $\mathcal{I}$, we define $M / J$ as the projection of $M$ on the space of matrices with null coefficients on the $I_{k}^{2}$ (see Figure 2.1):

$$
M / J=\left[(M / J)_{i j}\right]_{(i, j) \in \mathcal{I}^{2}}
$$

Copyright $\odot$ by SIAM. Unauthorized reproduction of this article is prohibited. 
with

$$
(M / J)_{i j}= \begin{cases}0 & \text { if }(i, j) \in I_{1}^{2} \cup \cdots \cup I_{K}^{2}, \\ m_{i j} & \text { if not. }\end{cases}
$$

If $\tau$ is a period on $\mathcal{I}$, we will write $M / \tau$ instead of $M /(\mathcal{I} / \tau)$.

2.2. Cellular coupler and cellular systems. In this section we build what we call cellular systems by way of a cellular coupler. Most past work in the field of synchronization deals with a specific way of coupling dynamical systems: one adds a quantity (that models interactions between subsystems) to the derivative of the systems. This leads to equations with the following typical shape (here, there are only two coupled systems):

$$
\begin{aligned}
& x_{1}^{\prime}(t)=F\left(x_{1}(t)\right)+G_{1}\left(x_{1}(t), x_{2}(t)\right), \\
& x_{2}^{\prime}(t)=F\left(x_{2}(t)\right)+G_{2}\left(x_{1}(t), x_{2}(t)\right) .
\end{aligned}
$$

The functions $G_{1}$ and $G_{2}$ are the coupling functions. The problem is then restated in terms of phase-shift variables, and efforts are made to detect stable states and to prove their stability.

Our approach is somewhat different. We study exclusively a way of coupling where the exchanges are made on the current state of the system. This means that the coupling quantity applies inside the map $F$, which leads us to the following type of equation:

$$
\begin{aligned}
& x_{1}^{\prime}(t)=F\left(x_{1}(t)+H_{1}\left(x_{1}(t), x_{2}(t)\right)\right), \\
& x_{2}^{\prime}(t)=F\left(x_{2}(t)+H_{2}\left(x_{1}(t), x_{2}(t)\right)\right) .
\end{aligned}
$$

Remark 2. We stress the point that those two different ways of handling coupled systems are quite equivalent in most cases. Indeed, starting with (2.1), as soon as $G_{1}$ and $G_{2}$ stay in the range of $F$ (which is likely if the coupling functions are small), we can rewrite them in the second form of (2.2) involving some functions $H_{1}$ and $H_{2}$.

The last kind of coupled system is sometimes studied (for instance, in [12]) but never broadly. (Indeed, if one wants some quantitative results about convergence of trajectories, one must work with specific equations and dynamical systems.) Even in a few papers that are quite general (as the very interesting [25]) some strong assumptions are made. (In [25] authors deal with symmetric periodic solutions). The kind of coupled systems we handle are a generalization of that described in (2.2.) Its general shape is

$$
x_{i}^{\prime}(t)=F_{i}\left(\sum_{j \in \mathcal{I}} c_{i j}\left(x_{i}(t)\right) x_{j}(t)\right) .
$$

Each cell $i \in \mathcal{I}$ owns its own differential system represented by a map $F_{i}$. Hence, all the dynamical systems are not forcibly identical; they don't even have the same shape. Moreover, we won't assume that they are weakly coupled (as in the classical paper of Winfree [27]). We simply assume that a cell $i$ "interprets" its own environment by mean of the functions $c_{i j}$.

Now, before giving the exact definition of a cellular coupler, we recall that $\mathcal{S}$ may be seen as a module on the ring $\prod_{i \in \mathcal{I}} \mathcal{L}\left(E_{i}\right) .(\mathcal{L}(A, B)$ is the space of continuous linear operators from $A$ to $B$, written $\mathcal{L}(A)$ if $A=B$.) Then, $\mathcal{L}(\mathcal{S})$ has to be understood as 
the space of continuous linear operators on $\mathcal{S}$ with coefficients in the spaces $\mathcal{L}\left(E_{i}, E_{j}\right)$. Any $M \in \mathcal{L}(\mathcal{S})$ may then be written as an infinite (if $\mathcal{I}$ isn't finite) matrix:

$$
M=\left[m_{i j}\right]_{(i, j) \in I^{2}}, \quad m_{i j} \in \mathcal{L}\left(E_{j}, E_{i}\right) .
$$

In this context, the definition of a cellular coupler on $\mathcal{I}$ is as follows.

Definition 2.1. A cellular coupling map on $\mathcal{I}$ is a map

$$
\begin{aligned}
c: \mathcal{S} & \longrightarrow \mathcal{L}(\mathcal{S}), \\
x & \longmapsto c(x)
\end{aligned}
$$

such that the matrix $\left[c_{i j}\right]_{(i, j) \in \mathcal{I}^{2}}$ satisfies the following:

1. $\forall(i, j) \in \mathcal{I}^{2}, \forall x \in \mathcal{S}, c_{i j}(x)$

depends only on $x_{i}$ (so that we may consider it as a map $c_{i j}: E_{i} \rightarrow \mathcal{L}\left(E_{j}, E_{i}\right)$ );

2. $\forall i \in \mathcal{I}, \forall x_{i} \in E_{i}, \sum_{j \in \mathcal{I}}\left\|c_{i j}\left(x_{i}\right)\right\|_{i}<+\infty$.

Then, $c$ defines a cellular coupler $\widetilde{c}$ on $\mathcal{I}$ in the following way:

$$
\begin{aligned}
\widetilde{c}: \mathcal{S} & \longrightarrow \mathcal{S}, \\
x & \longmapsto c(x) . x .
\end{aligned}
$$
$\widetilde{c}(x)$ :

We will sometimes use the convenient following notation for the components of

$$
\widetilde{c}(x)_{i}=c_{i}\left(x_{i}\right) \cdot x
$$

(as the $c_{i j}(x)$ depend only on $x_{i}$ ).

In other words (for the sake of simplicity, we consider only examples with a finite population), for any $x \in \mathcal{S}$, the matrix $c(x)$ has the following shape:

$$
c(x)=\left[\begin{array}{ccc}
c_{11}\left(x_{1}\right) & \cdots & c_{1 k}\left(x_{1}\right) \\
\vdots & \ddots & \vdots \\
c_{k 1}\left(x_{k}\right) & \cdots & c_{k k}\left(x_{k}\right)
\end{array}\right]=\left[\begin{array}{c}
c_{1}\left(x_{1}\right) \\
\vdots \\
c_{k}\left(x_{k}\right)
\end{array}\right] \in \mathcal{L}(\mathcal{S})
$$

Then

$$
\widetilde{c}(x)=c(x) \cdot x=\left[\begin{array}{c}
c_{11}\left(x_{1}\right) \cdot x_{1}+\cdots+c_{1 k}\left(x_{1}\right) \cdot x_{k} \\
\vdots \\
c_{k 1}\left(x_{k}\right) \cdot x_{1}+\cdots+c_{k k}\left(x_{k}\right) \cdot x_{k}
\end{array}\right]=\left[\begin{array}{c}
c_{1}\left(x_{1}\right) \cdot x \\
\vdots \\
c_{k}\left(x_{k}\right) \cdot x
\end{array}\right] \in \mathcal{S} .
$$

Remark 3. The second property in the previous definition insures a bounded convergence property on the $c_{i}$ in the following sense: let's choose $x_{i} \in E_{i}$ and $\left(y^{k}\right)_{k \in \mathbb{N}}$ a sequence in $\mathcal{S}_{\infty}$ that converges to $y \in \mathcal{S}_{\infty}$; then

$$
\lim _{k \rightarrow+\infty} c_{i}\left(x_{i}\right) \cdot y^{k}=c_{i}\left(x_{i}\right) \cdot y
$$

Moreover, we may also deduce that the $c_{i}$ are continuous on $E_{i}$ in the following way: if a sequence $\left(x_{i}^{k}\right)_{k \in \mathbb{N}}$ in $E_{i}$ converges to $x_{i} \in E_{i}$, then for any $y \in \mathcal{S}_{\infty}$

$$
\lim _{k \rightarrow+\infty} c_{i}\left(x_{i}^{k}\right) \cdot y=c_{i}\left(x_{i}\right) \cdot y
$$

Now we can define a cellular system, as follows.

Copyright (C) by SIAM. Unauthorized reproduction of this article is prohibited. 
DEFINITION 2.2. Let $F_{\mathcal{I}}$ be a vector field on $\mathcal{S}$ given by a family $\left\{F_{i}\right\}_{i \in \mathcal{I}}$ of vector fields on the $E_{i}$. Let $\widetilde{c}$ be a cellular coupler on $\mathcal{I}$. $\left(\mathcal{I}, F_{\mathcal{I}}, \widetilde{c}\right)$ is called a cellular system. A trajectory of this system is a trajectory $x \in \mathcal{T}$ that satisfies

$$
x^{\prime}=F_{\mathcal{I}} \circ \widetilde{c}(x)=F_{\mathcal{I}}(c(x) \cdot x) ;
$$

in other words,

$$
\begin{aligned}
\forall i \in \mathcal{I}, \forall t \in \Omega, \quad x_{i}^{\prime}(t) & =F_{i}\left(\sum_{j \in \mathcal{I}} c_{i j}\left(x_{i}(t)\right) \cdot x_{j}(t)\right) \\
& =F_{i}\left(c_{i}\left(x_{i}(t)\right) \cdot x(t)\right) .
\end{aligned}
$$

This equation may be naturally interpreted in biological terms: the cell $i$ behaves according to a mean of the states of all other cells $x_{j}$, but only its state defines how this mean is computed (the cell interprets its own environment), and this link state $\leftrightarrow$ interpreting function has no reason to be linear in $x_{i}$.

In the next section we start by exposing algebraic links between a cellular coupler and a component periodic trajectory, and then we turn to our localization lemma.

3. Localization lemma. The forthcoming result can be used in many ways and generalized as, for the sake of simplicity, we did not use the weakest assumptions under which it holds. (For example, the series convergence in the proof can be insured in many other contexts.)

Lemma 3.1. Let $\left(\mathcal{I}, F_{\mathcal{I}}, \widetilde{c}\right)$ be a cellular system and $\tau$ a period on $\mathcal{I}$. Let $U \subset \mathcal{S}$ on which $F_{\mathcal{I}}$ is injective. If $x \in \mathcal{T}^{\tau}$ is a $C P(\tau)$ trajectory of the cellular system that satisfies

1. $x(\Omega) \subset \mathcal{S}_{\infty}$,

2. $\widetilde{c}(x)(\Omega) \subset U$,

then there exists $b \in \mathcal{S}_{\infty}$ such that for any $t \in \Omega$

$$
x(t)-b \in \operatorname{ker}[c(x(t)) / \tau] .
$$

Remark 4. Note that the first condition on $x$ is useless if $\mathcal{I}$ is finite.

The previous result is not very practical as the right-hand side involves the trajectory $x$ itself, which is unknown. As there is no ambiguity, we define the kernel of $p_{\mathcal{I}(\tau)}(c)$ as

$$
\operatorname{ker}(c / \tau)=\bigcup_{x \in \mathcal{S}} \operatorname{ker}(c(x) / \tau) .
$$

Hence we may give a weaker version of the previous lemma.

COROLLARY 3.2. Under the conditions of Lemma 3.1 there exists $b \in \mathcal{S}$ such that

$$
x(\Omega)-b \in \operatorname{ker}(c / \tau) .
$$

Before laying out the proof, it may be interesting to explain how we'll use this result: let's suppose that a cellular system has a component periodic trajectory; if this trajectory is not component quasi-periodic, then the partition $\mathcal{I} / \tau$ is trivial, and $\operatorname{ker}(c / \tau)$ is the whole space $\mathcal{S}$. On the other hand, if this trajectory is component quasi-periodic, then $\mathcal{I} / \tau$ is not trivial and $\operatorname{ker}(c / \tau)$ may be smaller than $\mathcal{S}$. In the most 
illustrative case, $\operatorname{ker}(c / \tau)=\{0\}$, so that $x=b$ is a constant trajectory. (Let's recall that a $\mathrm{CP}(\tau)$ has no constant components.) This why we speak of localization. In the next section, among other things, we will study some simple cases where $\operatorname{ker}\left(p_{\mathcal{I}(\tau)}(c)\right)$ is small enough to insure us that there's no component quasi-periodic trajectory.

Proof of Lemma 3.1. First, let's check that $\widetilde{c}(x)$ is $\operatorname{CP}(\tau)$. For any $i \in \mathcal{I}, x_{i}^{\prime}$ is $\tau(i)$-periodic and nonconstant for $x_{i}$. Letting $U_{i}=U \cap E_{i}, F_{i}$ has to be injective on $U_{i}$. Hence, as $x$ is a trajectory of the cellular system, $F_{i}\left(\widetilde{c}(x)_{i}\right)$ must be periodic, and then $\widetilde{c}(x)_{i}$ is $\tau(i)$-periodic. Therefore, $\widetilde{c}(x)$ is $\operatorname{CP}(\tau)$.

Now, according to the partition $\mathcal{I}(\tau)=\left\{\mathcal{I}_{k}\right\}_{k \in K}$ defined by $\tau$ (see section 2.1), let $k \in K$ and $i \in \mathcal{I}_{k}$. For any $M \in \mathbb{N}$ we define the following set:

$$
\mathcal{I}_{k}^{M}=\mathcal{I}_{k} \cap \llbracket 0, M \rrbracket
$$

The set $\tau\left(\mathcal{I}_{k}^{M}\right)$ is now a finite dependent set, so that we can consider its $l c m \tau_{k}^{M}$. Now, for any $j \in \mathcal{I}_{k}^{M}, x_{j}$ and $\widetilde{c}(x)_{j}$ are $\tau_{j}^{M}$-periodic, so that, for any integer $N$,

$$
\begin{aligned}
& \widetilde{c}(x)_{i}(t)=\frac{1}{N+1} \sum_{l=0}^{N} \widetilde{c}(x)_{i}\left(t+l \tau_{k}^{M}\right) \\
& =\frac{1}{N+1} \sum_{l=0}^{N} c_{i}\left(x_{i}\left(t+l \tau_{k}^{M}\right)\right) \cdot x\left(t+l \tau_{k}^{M}\right) \\
& =\frac{1}{N+1} \sum_{l=0}^{N} c_{i}\left(x_{i}(t)\right) \cdot x\left(t+l \tau_{k}^{M}\right) \\
& =\frac{1}{N+1} \sum_{l=0}^{N} c_{i}\left(x_{i}(t)\right) \cdot\left[\mathbb{1}_{\mathcal{I}_{k}^{M}} \cdot x\left(t+l \tau_{k}^{M}\right)+\mathbb{1}_{\mathcal{I}_{k}-\mathcal{I}_{k}^{M}} \cdot x\left(t+l \tau_{k}^{M}\right)\right. \\
& +\mathbb{1}_{\left.\mathcal{C I}_{k}^{M} \cdot x\left(t+l \tau_{k}^{M}\right)\right]} \\
& =c_{i}\left(x_{i}(t)\right) \cdot\left[\mathbb{1}_{\mathcal{I}_{k}^{M}} \cdot x(t)+\frac{1}{N+1} \sum_{l=0}^{N}\left(\mathbb{1}_{\mathcal{I}_{k}-\mathcal{I}_{k}^{M}} \cdot x\left(t+l \tau_{k}^{M}\right)\right)\right. \\
& \left.+\frac{1}{N+1} \sum_{l=0}^{N}\left(\mathbb{1}_{\mathcal{C I}_{k}^{M}} \cdot x\left(t+l \tau_{k}^{M}\right)\right)\right] \\
& =c_{i}\left(x_{i}(t)\right) \cdot\left[\mathbb{1}_{\mathcal{I}_{k}^{M}} \cdot x(t)\right] c_{i}\left(x_{i}(t)\right) \cdot\left[\mathbb{1}_{\mathcal{I}_{k}-\mathcal{I}_{k}^{M}} \cdot\left(\frac{1}{N+1} \sum_{l=0}^{N} x_{j}\left(t+l \tau_{k}^{M}\right)\right)\right] \\
& +c_{i}\left(x_{i}(t)\right) \cdot\left[\mathbb{1}_{\complement \mathcal{I}_{k}} \cdot\left(\frac{1}{N+1} \sum_{l=0}^{N} x_{j}\left(t+l \tau_{k}^{M}\right)\right)\right] \text {. }
\end{aligned}
$$

From Remark 3 it's easy to show that one has the following limits for the two first lines of the previous equation:

$$
\begin{aligned}
\lim _{M \rightarrow+\infty} c_{i}\left(x_{i}(t)\right) \cdot\left[\mathbb{1}_{\mathcal{I}_{k}^{M}} \cdot x(t)\right]=c_{i}\left(x_{i}(t)\right) \cdot x(t) \\
\lim _{M, N \rightarrow+\infty} c_{i}\left(x_{i}(t)\right) \cdot\left[\mathbb{1}_{\mathcal{I}_{k}-\mathcal{I}_{k}^{M}} \cdot\left(\frac{1}{N+1} \sum_{l=0}^{N} x_{j}\left(t+l \tau_{k}^{M}\right)\right)\right]=0 .
\end{aligned}
$$

Copyright (C) by SIAM. Unauthorized reproduction of this article is prohibited. 
Now, regarding the last line, as for all $j \in \mathcal{C} \mathcal{I}_{k}, \tau_{k}^{M}$ and $\tau(j)$ are noncommensurable. If we denote by $\tau_{j}^{\prime}$ the minimal period of $x_{j}$ (generator of its group of periods), as $\tau(j)=n_{j} \tau_{j}^{\prime}$ for a certain integer $n_{j}, \tau_{k}^{M}$ and $\tau_{j}^{\prime}$ are also noncommensurable. Therefore, the sequence $\left(\frac{t+l \tau_{k}^{M}}{\tau_{j}^{\prime}}\right)_{l \in \mathbb{N}}$ is equidistributed $\bmod 1$, and we may apply some classic ergodic theorem (see, for instance, $[24,4]$ ) and write

$$
\lim _{N \rightarrow+\infty} \frac{1}{N+1} \sum_{l=0}^{N} x_{j}\left(t+l \tau_{k}^{M}\right)=\frac{1}{\tau_{j}^{\prime}} \int_{0}^{\tau(j)} x_{j}(s) d s=\frac{n_{j}}{\tau(j)} \int_{0}^{\tau(j)} x_{j}(s) d s .
$$

We can now define the state $b$ as

$$
b=\left[b_{j}\right]_{j \in \mathcal{I}}, \quad b_{j}=\frac{n_{j}}{\tau(j)} \int_{0}^{\tau(j)} x_{j}(s) d s .
$$

Applying Remark 3 once again, we find that

$$
\lim _{N \rightarrow+\infty} c_{i}\left(x_{i}(t)\right)\left[\mathbb{1}_{\mathfrak{C} \mathcal{I}_{k}} \cdot\left(\frac{1}{N+1} \sum_{l=0}^{N} x\left(t+l \tau_{k}^{M}\right)\right)\right]=c_{i}\left(x_{i}(t)\right)\left[\mathbb{1}_{\mathfrak{C} \mathcal{I}_{k}} . b\right] ;
$$

hence, we have shown that

$$
\widetilde{c}(x)_{i}(t)=c_{i}\left(x_{i}(t)\right) \cdot\left[\mathbb{1}_{\mathcal{I}_{k}^{M}} \cdot x(t)\right]+c_{i}\left(x_{i}(t)\right)\left[\mathbb{1}_{\mathcal{C I}_{k}} \cdot b\right] .
$$

However, obviously, from the beginning we had

$$
\widetilde{c}(x)_{i}(t)=c_{i}\left(x_{i}(t)\right) \cdot\left[\mathbb{1}_{\mathcal{I}_{k}^{M}} \cdot x(t)\right]+c_{i}\left(x_{i}(t)\right)\left[\mathbb{1}_{\mathcal{C I}_{k}} \cdot x(t)\right],
$$

so that

$$
c_{i}\left(x_{i}(t)\right)\left[\mathbb{1}_{\mathfrak{C} \mathcal{I}_{k}} \cdot x(t)\right]=c_{i}\left(x_{i}(t)\right)\left[\mathbb{1}_{\mathfrak{C} \mathcal{I}_{k}} \cdot b\right] .
$$

The previous work can be done for any $i$ which belongs to $\mathcal{I}_{k}$, and for any $k \in K$; hence we can conclude using our notation:

$$
(c(x(t)) / \tau)(x(t)-b)=0,
$$

which is exactly what we claimed.

In order to study the synchronization phenomenon, we need to extend the previous result to trajectories that converge to component (quasi-) periodic trajectories. The structure of the previous result and the way it's been proved make this extension quite easy, as follows.

Lemma 3.3. Let $\left(\mathcal{I}, F_{\mathcal{I}}, \widetilde{c}\right)$ be a cellular system and $\tau$ a period on $\mathcal{I}$. Let $U$ be a closed subset of $\mathcal{S}$ on which $F_{\mathcal{I}}$ is injective. Let $x$ be an aCP $(\tau)$ trajectory

$$
x=y+\alpha, \quad y \in \mathcal{T}^{\tau}, \quad \lim _{t \rightarrow+\infty} \alpha(t)=0
$$

such that

1. $x(\Omega) \subset \mathcal{S}_{\infty}$;

2. $\widetilde{c}(x)(\Omega) \subset U$;

3. $x^{\prime}$ is aCP $(\tau)$ (or equivalently: $\lim _{t \rightarrow+\infty} \alpha^{\prime}(t)=0$ ).

Copyright $@$ ( ) by SIAM. Unauthorized reproduction of this article is prohibited. 
Then there exists $b \in \mathcal{S}_{\infty}$ such that for any $t \in \Omega$

$$
y(t)-b \in \operatorname{ker}[c(y(t)) / \tau]
$$

and also

$$
y(t)-b \in \operatorname{ker}[c / \tau] .
$$

Proof. First, let's prove that $\widetilde{c}(x)$ is $\operatorname{aCP}(\tau)$. Letting $i \in \mathcal{I}$, as $x$ is a solution to the cellular system, one has

$$
x_{i}^{\prime}(t)=y_{i}^{\prime}(t)+\alpha_{i}^{\prime}(t)=F_{i}\left(\widetilde{c}(x(t))_{i}\right) .
$$

As $y_{i}$ is $\tau(i)$-periodic, $y_{i}^{\prime}$ is $\tau(i)$-periodic; hence, for any $l \in \mathbb{Z}$,

$$
y_{i}^{\prime}(t)+\alpha_{i}^{\prime}(t+l \tau(i))=F_{i}\left(\widetilde{c}(x(t+l \tau(i)))_{i}\right) .
$$

As $\alpha_{i}$ vanishes when $t \rightarrow+\infty$, we know that the right-hand side (rhs) has a limit when $t \rightarrow+\infty$. By hypothesis, $F_{i}$ is injective on $U_{i}$, which is a closed set. This insures that $x(t+l \tau(i))_{i}$ has a limit as $t \rightarrow+\infty$; we name this limit $z_{i}(t)$. Now, for any $k \in \mathbb{Z}$, one has

$$
y_{i}^{\prime}(t+k \tau(i))+\alpha_{i}^{\prime}(t+(l+k) \tau(i))=F_{i}\left(\widetilde{c}\left(x\left(t+k \tau_{i}+l \tau(i)\right)\right)_{i}\right),
$$

so that, letting $l \rightarrow+\infty$, we obtain

$$
y_{i}^{\prime}(t)=y_{i}^{\prime}(t+k \tau(i))=F_{i}\left(\widetilde{c}\left(z_{i}(t+k \tau(i))\right)_{i}\right) .
$$

As $F_{i}$ is injective, this proves that $z_{i}(t+k \tau(i))=z_{i}(t) ; z_{i}$ is then $\tau(i)$-periodic. Hence, one may write

$$
\widetilde{c}(x)=z(t)+\beta(t)
$$

where $z$ is $\mathrm{CP}(\tau)$ and $\lim _{t \rightarrow+\infty} \beta(t)=0$.

Now, we can write, if $i \in \mathcal{I}_{k}$ (for the sake of simplicity, we won't repeat the arguments involving some bounded $l \mathrm{~cm}$ used in the previous proof),

$$
\begin{aligned}
& \frac{1}{N+1} \sum_{l=0}^{N} \widetilde{c}(x)_{i}(t+l \tau(i)) \\
& =\frac{1}{N+1} \sum_{l=0}^{N} c_{i}\left(x_{i}(t+l \tau(i))\right) \cdot(x(t+l \tau(i))) \\
& =\frac{1}{N+1} \sum_{l=0}^{N} c_{i}\left(x_{i}(t+l \tau(i))\right) \cdot\left(y_{i}(t+l \tau(i))+\alpha_{i}(t+l \tau(i))\right) \\
& =\frac{1}{N+1} \sum_{l=0}^{N} c_{i}\left(x_{i}(t+l \tau(i))\right) \cdot\left(y_{i}(t+l \tau(i))\right)+o(1) \\
& =\frac{1}{N+1} \sum_{l=0}^{N} c_{i}\left(y_{i}(t)+\alpha_{i}(t+l \tau(i))\right) \cdot\left(\mathbb{1}_{\mathcal{I}_{k}} \cdot y_{i}(t)\right) \\
& \quad+\frac{1}{N+1} \sum_{l=0}^{N} c_{i}\left(y_{i}(t)+\alpha_{i}(t+l \tau(i))\right) \cdot\left(\mathbb{1}_{\mathbf{C} \mathcal{I}_{k}} \cdot y_{i}(t)\right)+o(1) .
\end{aligned}
$$

Copyright (c) by SIAM. Unauthorized reproduction of this article is prohibited. 
Using the last part of Remark 3 , as $\lim _{l \rightarrow+\infty} \alpha_{i}\left(t+l \tau_{i}\right)=0$ we have

$$
\begin{aligned}
& \frac{1}{N+1} \sum_{l=0}^{N} \widetilde{c}(x)_{i}(t+l \tau(i)) \\
& =\frac{1}{N+1} \sum_{l=0}^{N} c_{i}\left(y_{i}(t)\right) \cdot\left(\mathbb{1}_{\mathcal{I}_{k}} \cdot y_{i}(t)\right) \frac{1}{N+1} \sum_{l=0}^{N} c_{i}\left(y_{i}(t+l \tau(i))\right) \cdot\left(\mathbb{1}_{\mathbf{C} \mathcal{I}_{k}} \cdot y_{i}(t)\right)+o(1)
\end{aligned}
$$

and

$$
\lim _{N \rightarrow+\infty} \frac{1}{N+1} \sum_{l=0}^{N} \widetilde{c}(x)_{i}(t+l \tau(i))=\widetilde{c}(y)_{i}(t) .
$$

Using the same kind of arguments as in the previous proof, we find a vector $b \in \mathcal{S}_{\infty}$ satisfying

$$
\widetilde{c}(y)_{i}(t)=c_{i}\left(y_{i}(t)\right) \cdot\left[\mathbb{1}_{\mathcal{I}_{k}} \cdot y(t)+\mathbb{1}_{\mathcal{C I}_{k}} \cdot b\right],
$$

which leads to the conclusion.

This result may have an interesting interpretation: let $x$ be a solution of a cellular system that converges toward a quasi-periodic solution (i.e., $x$ is aCP $(\tau)$ and converges toward a nonperiodic $\mathrm{CP}(\tau)$ trajectory $y$ ). Let us assume that $\operatorname{ker}[c / \tau]=\{0\}$. (If we think of randomly chosen coupler, this is the generic case.) From what precedes we know that $y$ is, in fact, a constant solution, and the behavior of $x$ illustrates the oscillation death phenomenon (quenching). For example, in the case of coupled oscillators, if the natural frequencies of all oscillators mismatch in a way that only quasi-periodic solutions could occur, the couplers' properties may force this quenching phenomenon (ruling out those solutions from existing).

In the next section we give some examples of results based upon those lemmas.

\section{Applications.}

4.1. Simple examples of applications to classical differential or coupled systems. In this section, we give two examples of classical differential systems to which the cellular systems point of view may be applied. We start off with some Stuart-Landau oscillators.

4.1.1. Stuart-Landau oscillators. As this is the first example, for the sake of simplicity we consider a specific kind of coupling Stuart-Landau oscillators. Let us write the equations that drive an array of Stuart-Landau oscillators with nearest neighbor coupling:

$$
z_{j}^{\prime}=\left(G+i \omega_{j}\right) z_{j}-G\left|z_{j}\right|^{2} z_{j}+\kappa\left(z_{j}-z_{j+1}\right),
$$

where $N$ is the number of oscillators, $j \in\{1, \ldots, N\}(j=N+1$ means $j=1), z_{j}$ is a complex variable describing the state of the $j$ th oscillator, $G$ a parameter of nonlinear gain, $w_{j}$ the natural frequency, and $\kappa$ the coupling strength.

First, we want to give this system the shape of a cellular system. This is quite easily done with $\mathcal{I}=\{1, \ldots, N\}, E_{j}=\mathbb{C}$ (equivalently $E_{j}=\mathbb{R}^{2}$ ), and the coupler at 
point $z=\left(z_{1}, \ldots, z_{N}\right)$ :

$$
c(z)=\left[\begin{array}{cccccc}
a\left(z_{1}\right) & -\kappa & 0 & \cdots & 0 & -\kappa \\
-\kappa & a\left(z_{2}\right) & -\kappa & & & 0 \\
0 & -\kappa & \ddots & \ddots & & \vdots \\
\vdots & & \ddots & a\left(z_{j}\right) & \ddots & 0 \\
0 & & & \ddots & \ddots & -\kappa \\
-\kappa & 0 & \cdots & 0 & -\kappa & a\left(z_{N}\right)
\end{array}\right]
$$

with

$$
a(z)=G\left(1-|z|^{2}\right)+i \omega_{j}+\kappa .
$$

Now, let us assume that for a period $\tau$ on $\mathcal{I}, z$ is $\operatorname{CQP}(\tau)$. (Thus, each component of $z$ is periodic, but the set of all periods is not dependent.) We know from the localization lemma that there exists a constant state $b \in \mathcal{S}$ such that

$$
z(t)-b \in \operatorname{ker}[c(z(t)) / \tau] .
$$

Two different cases may occur:

- $\mathcal{I} / \tau=\{1, \ldots, N\}$ (none of the frequencies are commensurable). In that case one has

$$
c(z) / \tau=\left[\begin{array}{cccccc}
0 & -\kappa & 0 & \cdots & 0 & -\kappa \\
-\kappa & 0 & -\kappa & & & 0 \\
0 & -\kappa & \ddots & \ddots & & \vdots \\
\vdots & & \ddots & 0 & \ddots & 0 \\
0 & & & \ddots & \ddots & -\kappa \\
-\kappa & 0 & \cdots & 0 & -\kappa & 0
\end{array}\right],
$$

so that, for instance, $z_{1}=b_{1}+b_{2}-z_{2}$. This leads to a contradiction, as $z_{1}$ and $z_{2}$ would have the same period.

- $\mathcal{I} / \tau \neq\{1, \ldots, N\}$. In that case, at least two cells belong to the same class of the partition $\mathcal{I} / \tau$. In that case, it is easily seen that at least one line of $c(z) / \tau$ contains only one nonzero element $(-\kappa)$. Thus, there exists $k \in \mathcal{I}$ that satisfies

$$
z_{k}=-d ;
$$

again, this contradicts our assumptions about periodic motion (they must be nonconstant).

This quick analysis tells us that if every oscillator has a periodic behavior, all of them have a common frequency. There are many ways of coupling such an array of oscillators (see, for instance, [23]), but this example gives a sketch of what may be done in other cases: the discussion would depend only on the coupling matrix and the algebraic properties of all the different matrices $c / \tau$.

The next example shows why dealing with different Banach spaces $E_{i}$ may be useful: given an index $i$, if $x_{i}^{\prime}$ depends nonlinearly on $x_{j}, i$ and $j$ must belong to the same cell. This is why, in a general case, one may expect to handle very different spaces $E_{i}$. (In the worst case, there's only one big cell, and the system does not benefit from being written as a cellular system.) We enlighten this discussion with a really simple example. 
4.1.2. Coupled pendulum. If $E$ is a Banach space and $F$ a vector field on $E$, we want to see how this differential system may be written as a cellular system. For instance, one could consider a simple conservative system on $E=\mathbb{R}^{4}$ with a Hamilton equation given by (see [1])

$$
\begin{aligned}
x_{1}^{\prime} & =y_{1}, \\
y_{1}^{\prime} & =\alpha x_{1}-\beta x_{1}^{3}+\varepsilon x_{2}, \\
x_{2}^{\prime} & =y_{2}, \\
y_{2}^{\prime} & =-\gamma x_{2}+\varepsilon x_{1} .
\end{aligned}
$$

The first step is to identify the different cells of $\mathcal{I}$. We must factorize each term in the equations according to the different variables. For example, the second equation may be seen as

$$
y_{1}^{\prime}=\left(\alpha-\beta x_{1}^{2}\right) x_{1}+\varepsilon x_{2},
$$

so that the term $\left(\alpha-\beta x_{1}^{2}\right)$ has to be a part of the coupler we are building. Moreover, as it's the equation giving $y_{1}^{\prime}$, and as the way a cell computes how it interprets the population's state depends only on its own state, $x_{1}$ and $y_{1}$ have to belong to the same cell. In this simple example, the variables $x_{1}$ and $x_{2}$ are the only ones that must be gathered in the same cell. (The $x_{1}^{3}$ factor in the equation driving $y_{1}^{\prime}$ is not linear in $x_{1}$.) In the end, this leads to the following structure of a cellular system:

$$
\mathcal{I}=\{1,2,3\}
$$

with the Banach spaces

$$
E_{1}=\mathbb{R}^{2}, \quad E_{2}=E_{3}=\mathbb{R} .
$$

As should often be the case, the associated vector fields are just identity maps on $E_{i}$, and the coupler is then

$$
c=\left[\begin{array}{lll}
c_{11} & c_{12} & c_{13} \\
c_{21} & c_{22} & c_{23} \\
c_{31} & c_{32} & c_{33}
\end{array}\right]
$$

with

$$
\begin{aligned}
& c_{11}: \quad E_{1} \quad \longrightarrow \mathcal{L}\left(E_{1}\right), \\
& \left(x_{1}, y_{1}\right) \longmapsto\left[\begin{array}{cc}
0 & 1 \\
\alpha-\beta x_{1}^{2} & 0
\end{array}\right], \\
& c_{12}: E_{2} \longrightarrow \mathcal{L}\left(E_{2}, E_{1}\right), \\
& x_{2} \longmapsto\left[\begin{array}{l}
0 \\
\varepsilon
\end{array}\right] \text {, } \\
& c_{13}: E_{3} \quad \longrightarrow \quad \mathcal{L}\left(E_{3}, E_{1}\right), \\
& y_{2} \longmapsto\left[\begin{array}{l}
0 \\
0
\end{array}\right] \text {, } \\
& c_{21}: \quad E_{1} \longrightarrow \mathcal{L}\left(E_{1}, E_{2}\right), \\
& \left(x_{1}, y_{1}\right) \longmapsto\left[\begin{array}{ll}
0 & 0
\end{array}\right] \text {, }
\end{aligned}
$$

Copyright $@$ by SIAM. Unauthorized reproduction of this article is prohibited. 


$$
\begin{aligned}
c_{22}: E_{2} & \longrightarrow \mathcal{L}\left(E_{2}\right), \\
x_{2} & \longmapsto[0], \\
c_{23}: E_{3} & \longrightarrow \mathcal{L}\left(E_{3}, E_{2}\right), \\
y_{2} & \longmapsto[1], \\
c_{31}: \quad E_{1} & \longrightarrow \mathcal{L}\left(E_{1}, E_{3}\right), \\
\left(x_{1}, y_{1}\right) & \longmapsto\left[\begin{array}{cc}
\varepsilon & 0
\end{array}\right], \\
c_{32}: E_{2} & \longrightarrow \mathcal{L}\left(E_{2}, E_{3}\right), \\
x_{2} & \longmapsto[-\gamma], \\
c_{33}: E_{3} & \longrightarrow \mathcal{L}\left(E_{3}\right), \\
y_{2} & \longmapsto[0] .
\end{aligned}
$$

Now, before applying some of the previous techniques, we may compute the different decomposition of $c$ upon different nontrivial partitions of $\mathcal{I}$. Those partitions are

$$
\begin{gathered}
P_{1}=\{\{1\},\{2\},\{3\}\}, \quad P_{2}=\{\{1,2\},\{3\}\}, \\
P_{3}=\{\{1,3\},\{2\}\}, \quad P_{4}=\{\{1\},\{2,3\}\},
\end{gathered}
$$

which gives (in order to avoid any confusion, we write as $0_{i j}$ the $i \times j$ null matrix)

$$
\begin{array}{ccc}
c / P_{1}=\left[\begin{array}{ccc}
0_{22} & c_{12} & c_{13} \\
c_{21} & 0 & c_{23} \\
c_{31} & c_{32} & 0
\end{array}\right], & c / P_{2}=\left[\begin{array}{ccc}
0_{22} & 0_{21} & c_{13} \\
0_{12} & 0 & c_{23} \\
c_{31} & c_{32} & 0
\end{array}\right], \\
c / P_{3}=\left[\begin{array}{ccc}
0_{22} & c_{12} & 0_{21} \\
c_{21} & 0 & c_{23} \\
0_{12} & c_{32} & 0
\end{array}\right], & c / P_{4}=\left[\begin{array}{ccc}
0_{22} & c_{12} & c_{13} \\
c_{21} & 0 & 0 \\
c_{31} & 0 & 0
\end{array}\right] .
\end{array}
$$

Now, in order to simplify, we replace the $c_{i j}$ 's that are identically zero by 0 , obtaining the following different matrices:

$$
\begin{array}{lcc}
c / P_{1}=\left[\begin{array}{ccc}
0_{22} & c_{12} & 0_{21} \\
0_{12} & 0 & c_{23} \\
c_{31} & c_{32} & 0
\end{array}\right], & c / P_{2}=\left[\begin{array}{ccc}
0_{22} & 0_{21} & 0_{21} \\
0_{12} & 0 & c_{23} \\
c_{31} & c_{32} & 0
\end{array}\right], \\
c / P_{3}=\left[\begin{array}{ccc}
0 & c_{12} & 0_{21} \\
0_{12} & 0 & c_{23} \\
0_{12} & c_{32} & 0
\end{array}\right], & c / P_{4}=\left[\begin{array}{ccc}
0_{22} & c_{12} & 0_{21} \\
0_{12} & 0 & 0 \\
c_{31} & 0 & 0
\end{array}\right] .
\end{array}
$$

In the end, writing the coupler as an application from $\mathcal{S}$ to $\mathcal{L}(\mathcal{S})$, one finds these four different $4 \times 4$ matrices (the 1 in the dimensions comes from the fact that the dimension of the first cell $P_{1}$ is 2):

$$
\begin{aligned}
& {\left[\begin{array}{cccc}
0 & 0 & 0 & 0 \\
0 & 0 & \varepsilon & 0 \\
0 & 0 & 0 & 1 \\
\varepsilon & 0 & -\gamma & 0
\end{array}\right],\left[\begin{array}{cccc}
0 & 0 & 0 & 0 \\
0 & 0 & 0 & 0 \\
0 & 0 & 0 & 1 \\
\varepsilon & 0 & -\gamma & 0
\end{array}\right],} \\
& {\left[\begin{array}{cccc}
0 & 0 & 0 & 0 \\
0 & 0 & \varepsilon & 0 \\
0 & 0 & 0 & 1 \\
0 & 0 & -\gamma & 0
\end{array}\right],\left[\begin{array}{llll}
0 & 0 & 0 & 0 \\
0 & 0 & \varepsilon & 0 \\
0 & 0 & 0 & 0 \\
\varepsilon & 0 & 0 & 0
\end{array}\right]}
\end{aligned}
$$

Copyright $\odot$ by SIAM. Unauthorized reproduction of this article is prohibited. 
At this point, we just have to check that the coupler has some good injectivity properties:

$$
\begin{aligned}
& \operatorname{ker}\left(c / P_{1}\right) \cap E_{2}=\operatorname{ker}\left(c / P_{4}\right) \cap E_{2}=\{0\}, \\
& \operatorname{ker}\left(c / P_{2}\right) \cap E_{3}=\operatorname{ker}\left(c / P_{3}\right) \cap E_{3}=\{0\} .
\end{aligned}
$$

So, we may apply Lemma 3.1 and, without any analytic calculus, state that this differential system may not admit any component quasi-periodic solution (unless one of the components would be a constant function). In other words, in case there exists a component periodic trajectory, it must be (globally) periodic.

Remark 5. As we focus mainly on the frequency locking phenomenon, the existence of a quasi-periodic solution is not the main topic here. That's why we must stress the point that this technique does not completely solve the problem of the existence of quasi-periodic solutions. Indeed, one must check (by other techniques) that there's no quasi-periodic solution which isn't component periodic. More precisely, one often wants to predict chaotic motions when coupling oscillators (see, for instance, [1]). What precedes could be of a certain help in an elimination procedure: once one knows that there is no CQP motion, ruling out, with other techniques, the existence of other kinds of solutions (such as the periodic solutions), one could predict chaos arising under certain conditions.

Before presenting some more general results, it seems interesting to show that our conclusions may hold in a more general case where the $c_{i j}$ are less simple.

Proposition 4.1 (generalized coupled pendulum). Let's consider a differential system which is driven by the following equations (we don't rename the functions, e.g., $\gamma$, that come from the previous model):

$$
\begin{aligned}
x_{1}^{\prime} & =a_{1}\left(x_{1}, y_{1}\right) x_{1}+a_{2}\left(x_{1}, y_{1}\right) y_{1}+a_{3}\left(x_{1}, y_{1}\right) x_{2}+a_{4}\left(x_{1}, y_{1}\right) y_{2}, \\
y_{1}^{\prime} & =a_{5}\left(x_{1}, y_{1}\right) x_{1}+a_{6}\left(x_{1}, y_{1}\right) y_{1} \\
x_{2}^{\prime} & =a_{7}\left(x_{2}\right) x_{2}+u\left(x_{2}\right) y_{2} \\
y_{2}^{\prime} & =\varepsilon\left(y_{2}\right) x_{1}+a_{8}\left(y_{2}\right) y_{1}-\gamma\left(y_{2}\right) x_{2}+a_{9}\left(y_{2}\right) y_{2} .
\end{aligned}
$$

If the maps $u$ and $\varepsilon$ never vanish, then the system has no component quasi-periodic solution.

This result does not have to be deep in itself, neither does it have to be the most general one we could have deduced from the previous discussion. It's just a sketch of how one can handle some structural properties of a differential system, applying Lemma 3.1, without going into deep and specific calculus.

The next section deals with less specific but more theoretical examples.

4.2. Some general examples of applications. The next example shows how some topological properties of a coupler (how it connects cells together) may influence the frequency locking phenomenon.

4.2.1. Chained cellular system. In this section, for the sake of simplicity, all the vector spaces $E_{i}$ have finite dimension.

We first study the case of differential systems for which the spaces $E_{i}$ have the same dimension and are coupled with $k$-nearest neighbors. (The finite dimension condition isn't necessary, but it makes the presentation simpler.) This case is formally described by a cellular system $\left(\mathcal{I}, F_{\mathcal{I}}, \widetilde{c}\right)$, where $\mathcal{I}$ is countable, all $\operatorname{dim}\left(E_{i}\right)=n$, and $\widetilde{c}$ satisfies

$$
\forall i, j \in \mathcal{I}, \quad|j-i|>k \Rightarrow c_{i j}=0
$$


This is what we call a chained cellular system. Adding the following condition on the coupler, we may reach a general result.

Definition 4.2. A cellular coupler $\widetilde{c}$ is said to have full rank if for any $i, j \in \mathcal{I}$ and $x \in \mathcal{S}$ the map $c_{i j}(x)$ has full rank.

Proposition 4.3. Let $\left(\mathcal{I}, F_{\mathcal{I}}, \widetilde{c}\right)$ be a chained cellular system coupled with $k$ nearest neighbors (all $E_{i}$ having the same finite dimension). Let $F_{\mathcal{I}}$ be injective on $U \subset \mathcal{S}$ and $x$ a $C P(\tau)$ trajectory that stays in $U$ (or aCP $(\tau)$ if $U$ is closed). If $\widetilde{c}$ has maximal rank and if there exists $I \in \mathcal{I} / \tau$ which contains $2 k$ consecutive cells, i.e., there exists $i \in \mathcal{I}$ such that

$$
\llbracket i, i+2 k-1 \rrbracket \subset I,
$$

then $\mathcal{I} / \tau=\{\mathcal{I}\}$ (equivalently, $\tau(\mathcal{I})$ is a dependent set).

Proof. Suppose that $I \neq \mathcal{I}$. There must exist $\llbracket i, i+2 k \rrbracket \subset I$ such that $i-1 \notin$ $I$. Then, line $i+k-1$ of the matrix $c(x(t)) / \tau$ contains only one nonzero element $c_{i+k-1, i-1}$. As this linear map is injective for any $t \in \Omega$, we know that

$$
\operatorname{ker}(c(x(t)) / \tau) \bigcap E_{i-1}=\{0\} .
$$

Applying Lemma 3.1, we know that there exists $b_{i-1} \in E_{i-1}$ such that for any $t \in \Omega$

$$
x_{i-1}(t)-b_{i-1} \in \operatorname{ker}(c(x(t)) / \tau) \bigcap E_{i-1} ;
$$

i.e., $x_{i-1}(t)=b_{i-1}$ is a constant map, which contradicts the definition of a component periodic trajectory. So we can conclude that $I=\mathcal{I}$.

If we assume that $\tau$ is bounded, this result may be restated as: "as soon as $k$ consecutive cells are synchronized (locked frequencies), then all the population is synchronized."

Moreover, we may drop some assumptions made on the common dimension of the $E_{i}$ and reach an interesting connecting result concerning the case $k=1$ (analogous to the the example of Stuart-Landau oscillators).

Proposition 4.4. Let $\left(\mathcal{I}, F_{\mathcal{I}}, \widetilde{c}\right)$ be a chained cellular system coupled with 1 nearest neighbor. Let $F_{\mathcal{I}}$ be injective on $U \subset \mathcal{S}$, and $x$ a $C P(\tau)$ trajectory that stays in $U$ (or aCP $(\tau)$ if $U$ is closed). If $\widetilde{c}$ has maximal rank and if there exist two sets $I_{1}$ and $I_{2}$ in $\mathcal{I} / \tau$ such that for $i \in \mathcal{I}$

$$
\llbracket i, i+1 \rrbracket \subset I_{1}, \quad \llbracket i+2, i+3 \rrbracket \subset I_{2},
$$

then $I_{1}=I_{2}$.

Proof. Suppose that the cells of $I_{1}$ have periods noncommensurable with those of $I_{2}$ (i.e., $I_{1} \neq I_{2}$ ). Following the previous proof, we know that the lines $i+1$ and $i+2$ of the matrix $c(x(t)) / \tau$ contains only one nonzero element, respectively $c_{i+1, i+2}$ and $c_{i+2, i+1}$. But, we recall that for any $t \in \Omega$

$$
c_{i+1, i+2}\left(x_{i+1}(t)\right): E_{i+2} \rightarrow E_{i+1}
$$

and

$$
c_{i+2, i+1}\left(x_{i+2}(t)\right): E_{i+1} \rightarrow E_{i+2} .
$$

As the coupler has maximal rank, one of the previous maps must be injective $\forall t \in \Omega$. Using the same argument as in the previous proof, we may conclude that either $x_{i+1}$ or $x_{i+2}$ is a constant map, both cases leading to a contradiction.

For the next example, we add some regularity conditions on the cellular system, which lead to an interesting description of $\mathcal{S}$. 
4.2.2. Localization results with bounded states. As $\left(\mathcal{S}_{\infty},\|\cdot\|_{\infty}\right)$ is a Banach space, the classic Picard-Lindelöf theorem is valid, and we can give a version adapted to cellular systems.

Proposition 4.5. If $F_{\mathcal{I}}: \mathcal{S}_{\infty} \rightarrow \mathcal{S}_{\infty}$ and $\widetilde{c}$ are locally Lipschitz, which is the case if for any $x \in \mathcal{S}_{\infty}$ there are a neighborhood $V=\prod_{i \in \mathcal{I}} V_{i}$, a positive number $k$, and a sequence $\left(k_{j}\right)_{j \in \mathcal{I}}$ of positive numbers such that

1. $\forall y, z \in V, \forall i \in \mathcal{I},\left\|F_{i}\left(y_{i}\right)-F_{i}\left(z_{i}\right)\right\|_{i} \leq k\left\|y_{i}-z_{i}\right\|_{i}$;

2. $\forall y, z \in V, \forall i \in \mathcal{I},\left\|c_{i j}\left(y_{i}\right)-c_{i j}\left(z_{i}\right)\right\|_{\left(E_{j}, E_{i}\right)} \leq k_{j}\left\|y_{i}-z_{i}\right\|_{i}$;

3. $\sum_{j \in \mathcal{I}} k_{j}<+\infty$

then, given any initial condition $\left(t^{0}, x^{0}\right)$ in $\mathbb{R} \times \mathcal{S}_{\infty}$, the cellular coupling admits a unique maximal solution $x$ that satisfies $x\left(t^{0}\right)=x^{0}$.

Before stating our localization result, we need to define the sets that any component quasi-periodic trajectory of the cellular system must avoid.

DEFINITION 4.6. Let $\widetilde{c}$ be a cellular coupler on $\mathcal{I}$. The set of regular points for $\widetilde{c}$ is defined as

$$
R(\widetilde{c})=\{x \in \mathcal{S}, \forall J \text { nontrivial partition of } \mathcal{I}, c(x) / J \text { is injective }\} .
$$

We say that $\widetilde{c}$ is regular if $R(\widetilde{c})=\mathcal{S}$.

Proposition 4.7. Under the conditions of Lemma 3.1 and Proposition 4.5, if there exists an infinite compact subset $V \subset \Omega$ such that

$$
\forall t \in V, \quad x(t) \in R(\widetilde{c}),
$$

then $\tau(\mathcal{I})$ is a dependent set.

One can rewrite this result in terms of differential systems, as follows.

Proposition 4.8. Under the conditions of Lemma 3.1 and Proposition 4.5, and if $\tau$ is bounded, a $C Q P(\tau)$ trajectory must "avoid" $R(\widetilde{c})$. (It can't cross this set on an infinite compact subset of $\Omega$.)

Proof of Proposition 4.7. Let suppose that $\mathcal{I} / \tau$ is not trivial. Applying Lemma 3.1, we know that

$$
c(x(t)) / \tau .(x(t)-b)=0 .
$$

The assumptions made on $\widetilde{c}$ ensure that

$$
\forall t \in V, \quad x(t)=b .
$$

As $V$ has an accumulation point, we may conclude that there exists $t_{0} \in V$ such that

$$
x^{\prime}\left(t_{0}\right)=0 .
$$

Proposition 4.5 may be applied; hence we know that $t \mapsto x(t)$ is a constant map, which contradicts the definition of a component periodic trajectory.

5. Conclusion. In this work we have built a general framework of cellular systems in order to handle a wide variety of coupled systems, and therefore a wide class of complex systems. We focused on an emergent property of those dynamical systems: the frequency locking phenomenon. Usually one observes solutions of particular coupled systems and shows that within suitable conditions synchronization must occur. Those results are qualitatively dependent on the systems of interest and do not stand in the general cases. We tried to change our point of view and to bring out completing 
results. We don't prove that synchronization ultimately happens, but instead we consider the problem at its end: if one supposes that some coupled systems converge to some oscillating behaviors, then they must be synchronized (frequencies are locked), regardless of the individual dynamical systems (as soon as the maps which define each of them are injective near the trajectories). In most papers (see, for instance, [13]) the population of coupled systems is implicitly defined and has only two cells (sometimes a finite number $N$, and more rarely an infinity). Moreover, in contrast to what most studies about synchronization issues state, we do not assume anything concerning the cell dynamics. (In particular, we don't assume that they are oscillators.) We assume only that they (asymptotically) exhibit periodic behaviors under the coupling effects. (The first assumption implies the second, but the opposite is clearly false.) In that sense, this technique could be used before any specific analysis, to rule out the existence of certain behaviors.

We believe that this way of reaching general results about cellular systems gives some explanations about why the frequency locking phenomenon emerges naturally in a large variety of coupled dynamical systems. Our results show that the following alternative is natural in many cases: either the whole population is synchronized, or its cells can't all have periodic behaviors.

Another interesting perspective is to apply this strategy to differential systems, as we outlined in the beginning of the fourth section. For example, in contrast to what happens in the general case of Hamiltonian systems, where limit tori are generally filled with quasi-periodic trajectories (especially after perturbations), our results suggest that concerning cellular systems, limit tori are mainly filled with periodic trajectories.

Moreover, we have achieved some similar work on a natural generalization of this strategy to noncountable population. (In order to model natural systems, it's often necessary to handle continuous populations.) We truly think that all these results are only a part of what can be done using cellular systems and that this work enlarges the possibilities for studying synchronization issues in some biologically inspired systems. But the scope of those kinds of cellular systems may be beyond synchronization questions, as it's quite general and allows some theoretical studies. Our approach could be a promising theoretical tool to model complex systems.

\section{REFERENCES}

[1] A. C. J. Luo, Predictions of quasi-periodic and chaotic motions in nonlinear Hamiltonian systems, Chaos Solitons Fractals, 28 (2006), pp. 627-649.

[2] M. Bennett, M. F. Schatz, H. Rockwood, and K. Wiesenfeld, Huygens's clocks, Proc. R. Soc. Lond., 458 (2002), pp. 563-579.

[3] J. Buck, Synchronous rhythmic flashing of fireflies. ii, Quart. Rev. Biol., 63 (1988), pp. 265289.

[4] L. Bunimovich, Dynamical Systems, Ergodic Theory and Applications, 2nd ed., Encyclopaedia Math. Sci. 100, Springer, New York, 2000.

[5] G. B. Ermentrout And W. C. Troy, Phaselocking in a reaction-diffusion system with a linear frequency gradient, SIAM J. Appl. Math., 46 (1986), pp. 359-367.

[6] L. Gaubert, Auto-organisation et émergence dans les systèmes couplés, individuation de données issues de systèmes biologiques couplés, Ph.D. thesis, Applied Mathematics, Université de Bretagne Occidentale, Brest, France, 2007.

[7] J.-P. Goedgebuer, P. Levy, and L. Larger, Laser cryptography by optical chaos, in Optical Information, Data Processing and Storage, and Laser Communication Technologies, Proc. SPIE 5135, J.-P. Goedgebuer, N. N. Rozanov, S. K. Turitsyn, A. S. Akhmanov, and V. Y. Panchenko, eds., 2003, pp. 14-20.

[8] P. Goel And B. Ermentrout, Synchrony, stability, and firing patterns in pulse-coupled oscillators, Phys. D, 163 (2002), pp. 191-216. 
[9] D. Gonze, J. Halloy, And A. Goldbeter, Stochastic models for circadian oscillations: Emergence of a biological rhythm, Int. J. Quantum Chem., 98 (2004), pp. 228-238.

[10] F. C. Hoppensteadt And J. B. Keller, Synchronization of periodical cicada emergences, Science, 194 (1976), pp. 335-337.

[11] C. Huygens, Horologium oscillatorium sive De motu pendulorum ad horologia aptato demonstrationes geometricae, F. Muguet, Paris, France, 1673.

[12] E. M. Izhikevich And F. C. Hoppensteadt, Slowly coupled oscillators: Phase dynamics and synchronization, SIAM J. Appl. Math., 63 (2003), pp. 1935-1953.

[13] N. Kopell And G. B. Ermentrout, Mechanisms of phase-locking and frequency control in pairs of coupled neural oscillators, in Handbook of Dynamical Systems II: Toward Applications, Elsevier, Amsterdam, 2002, pp. 3-54.

[14] Y. KuRAmoto, Self-entrainment of a population of coupled non-linear oscillators, in Proceedings of the International Symposium on Mathematical Problems in Theoretical Physics, Lcture Notes in Phys. 39, Springer, Berlin, Heidelberg, 1975, pp. 420-422.

[15] Y. Kuramoto, Chemical Oscillations, Waves, and Turbulence, Springer-Verlag, New York, Berlin, 1984 .

[16] S. C. Manrubia, A. S. Mikhailov, and D. H. Zanette, Emergence of Dynamical Order. Synchronization Phenomena in Complex Systems, World Scientific, Singapore, 2004.

[17] D. C. Michaels, E. P. Matyas, And J. Jalife, Mechanisms of sinoatrial pacemaker synchronization: A new hypothesis, Circulation Res., 61 (1987), pp. 704-714.

[18] L. M. Pecora, T. L. Carroll, G. A. Johnson, D. J. Mar, and J. F. Heagy, Fundamentals of synchronization in chaotic systems, concepts, and applications, Chaos, 7 (1997), pp. 520-543.

[19] A. Pikovsky, M. Rosenblumand, and J. Kurths, Synchronization: A Universal Concept in Nonlinear Sciences, Cambridge University Press, Cambridge, UK, 2001.

[20] I. Prigogine, Introduction to Thermodynamics of Irreversible Processes, Wiley, New York, 1967.

[21] I. Prigogine, Structure, dissipation, and life, in Theoretical Physics and Biology, M. Marois, North-Holland, Amsterdam, 1969, pp. 23-52.

[22] L. Ren And G. B. Ermentrout, Phase locking in chains of multiple-coupled oscillators, Phys. D, 143 (2000), pp. 56-73.

[23] N. F. Rulkov, L. Tsimring, M. G. Larsen, and M. Gabbay, Synchronization and beam forming in an array of repulsively coupled oscillators, Phys. Rev. E, 74 (2006), paper 056205.

[24] Y. G. SinAI, Introduction to Ergodic Theory, Princeton University Press, Princeton, NJ, 1976.

[25] I. Stewart and M. Golubitsky, Patterns of oscillation in coupled cell systems, in Geometry, Dynamics and Mechanics: 60th Birthday Volume for J. E. Marsden, P. P. Holmes and A. Weinstein, eds., Springer-Verlag, New York, 2002, pp. 243-286.

[26] S. Strogatz, Sync: The Emerging Science of Spontaneous Order, Hyperion, New York, 2003.

[27] A. Winfree, Biological rhythms and the behavior of populations of coupled oscillators, J. Theoret. Biol., 16 (1967), pp. 15-42.

[28] A. Winfree, The Geometry of Biological Time, Springer-Verlag, New York, 1990.

[29] C. Wu And L. O. ChuA, A Unified Framework for Synchronization and Control of Dynamical Systems, Technical Report UCB/ERL M94/28, EECS Department, University of California, Berkeley, Berkeley, CA, 1994.

Copyright (C) by SIAM. Unauthorized reproduction of this article is prohibited. 